\title{
PORTAL HYPERTENSION AND ITS RADIOLOGICAL INVESTIGATION
}

\author{
J. H. Middlemiss, M.D., F.F.R., D.M.R.D. \\ F. G. M. Ross, M.B., B.Ch., B.A.O., F.F.R., D.M.R.D.
}

From the Department of Radiodiagnosis, United Bristol Hospitals

PORTAL hypertension is a condition in which there is an abnormally high blood pressure in the portal system of veins which eventually leads to splenomegaly and in chronic cases, to hæmatemesis and melæna.

The portal circulation is unique in that it exists between two sets of capillaries, i.e. the capillaries of the spleen, pancreas, gall-bladder and most of the gastro-intestinal tract on the one hand and the sinusoids of the liver on the other hand. The liver parallels the lungs in that it receives a blood supply from an arterial source, the hepatic artery, and a venous source, the portal vein.

\section{The Portal Circulation}

Blood passes from the splanchnic arteries into the spleen, pancreas, gall-bladder, stomach and intestines from branches of the abdominal aorta. From the sinuses in the splenic pulp, the blood is collected into several tributaries which unite at the hilum of the spleen to form the splenic vein, which runs to the right along the upper posterior aspect of the pancreas to the region of the neck of the pancreas. As it crosses the upper abdomen, it receives as tributaries the short gastric veins, the left gastro-epiploic, pancreatic, inferior mesenteric and left gastric veins. The superior mesenteric vein is formed in the root of the mesentery from union of the jejunal and ileal veins, the ileo-colic, right and middle colic and inferior pancreatico-duodenal veins. The portal vein usually forms behind the neck of the pancreas, but occasionally in front of the pancreas, by the union of the superior mesenteric and splenic veins at the level of the body of the second lumbar vertebra. The portal vein runs upwards and to the right, but occasionally horizontally, in the free edge of the lesser omentum, behind the duodenal bulb and common bile duct, to terminate at the porta hepatis where it divides into two and sometimes three branches, which pass into the liver. The terminations of the left gastric and inferior mesenteric veins are variable. In two-fifths of persons they enter the splenic vein; in a further two-fifths they enter the portal and superior mesenteric veins respectively, and in the remaining one-fifth the left gastric vein enters the junction of the portal and splenic veins and the inferior mesenteric the junction of the superior mesenteric and splenic veins. In about $90 \%$ of people several veins enter the portal vein including the pyloric vein on the left side and the cystic and superior pancreaticoduodenal veins on the right side. Para-umbilical veins from the falciform ligament usually drain into the left branch of the portal vein but may drain into the right branch.

Small veins which are present on the serosal surface of the liver and in the surrounding peritoneal folds draining the diaphragm and stomach are known as accessory portal veins. They may unite with the portal vein or enter the liver independently.

The hepatic artery arises normally from the cœliac axis but it may arise as a separate trunk from the aorta. It runs upwards and to the right and divides into a left and right branch before entering the liver at the porta hepatis.

The venous return starts as small thin-walled branches in the centre of the lobules in the liver. They join interlobular branches which coalesce and finally unite to form two or three hepatic veins which enter the inferior vena cava just before it terminates in the right auricle.

The liver lobules each have a small hepatic vein in the centre and in defined areas round the periphery there are portal tracts each of which contains a radicle of the portal vein, hepatic artery and a small bile duct. The hepatic cells are arranged in sheets which are traversed by the sinusoids so that one-cell-thick plates of hepatic cells are formed. These plates form an irregular sponge which surrounds spaces or lacunæ in which the sinusoids are suspended by connective tissue fibrils. Between individual hepatic cells are bile canaliculi from which bile passes to collecting ductules which run to the portal tracts. Here small bile ducts are formed which drain eventually into the extra-hepatic bile ducts.

The sinusoids are thus modified capillaries lined with endothelial cells on a basement membrane and some of their cells are specially adapted reticulo-endothelial cells. known as Kupffer cells. The sinusoids form a honeycomb or 'hepatic labyrinth' in the lobules through which blood flows from the peripheral portal vein to the central hepatic vein. The hepatic artery divides into fine branches in the portal tracts which form a capillary network that drains into the portal vein and peripheral sinusoids. Some hepatic artery blood enters the sinusoids direct and it has been shown histologically that these small hepatic arterioles contain muscle pads and ring muscle in their walls which presumably control the hepatic artery blood flow. The principle of the lobule as a structural unit involves complete separation of the portal and hepatic veins, the terminal branches of which interdigitate with lobular parenchyma interposed in all places between them. However, anastomoses between them have been claimed and are supposed to be open in the absence of digestion.

The liver is surrounded by the hepatic capsule from which a connective tissue stroma extends into the portal tracts and the central canals. Within the lobule, radial collagenous fibres connect the central part of the lobule to the periphery and a fine reticulum supports the 
sinusoids. On the surface of the liver interlobular connective tissue unites with the capsule and through this connection capsular vessels communicate with the portal vein but not the hepatic vein.

The total liver blood flow is about $1,500 \mathrm{ml} . / \mathrm{min}$. in adults, of which about $80 \%$ is contributed by the portal vein. The liver blood flow can be measured by the bromsulphthalein excretion method or by radio-active isotopes.

The portal pressure can be measured at laparotomy by connecting a saline manometer to a cannula inserted into an unobstructed vein of the portal system: the fact that the abdomen is open probably makes very little difference to the reading. It has been shown, however, that the intrasplenic pressure is a relatively accurate indication of the portal venous pressure, though naturally it is slightly higher than the pressure in the portal vein itself. This is estimated by percutaneous needle puncture, usually in combination with trans-splenic portal venography. Another indirect method is to estimate the wedged hepatic vein pressure by passing a cardiac catheter through the right auricle into the inferior vena cava and thence into an hepatic vein. The catheter tip is passed out into the liver so that its external circumference blocks the internal circumference of an hepatic vein.

Normal portal pressures are:

$\begin{array}{llrl}\text { Intrasplenic } & \ldots & 3 \text { to } 17 \mathrm{~mm} . \mathrm{Hg} \\ \text { Portal vein } & \ldots & 7.5 \text { to } 16 \quad, \\ \text { Sinusoidal } & \ldots & 6 \text { to } 8,\end{array}$

Temporary occlusion of the portal vein in normal persons causes the portal venous pressure to rise immediately to 40 to $50 \mathrm{~mm}$. $\mathrm{Hg}$ and it remains at this level so long as the obstruction is maintained.

\section{Portal Hypertension}

The portal venous pressure may be raised as a result of increased inflow through the splanchnic arteries or due to increased resistance to the outflow from the portal system of veins. Increased inflow is only likely to be significant if the inflow resistance is diminished, which occurs in cases of splenic artery-vein fistulæ. Increased resistance to the outflow of the portal venous blood is caused by $(a)$ conditions which raise the hepatic venous pressure (post - or supra-hepatic obstruction), $(b)$ lesions of the liver itself (intra-hepatic obstruction), and (c) lesions affecting the portal and splenic veins (extra-hepatic obstruction).

\section{(a) Supra-hepatic Obstruction}

This will occur in congestive heart failure and constrictive pericarditis. If the portal hypertension is secondary to a generalized rise in venous pressure, it will be of no consequence clinically as a collateral circulation will only develop if there is a pressure gradient between the portal and systemic veins. In veno-occlusive disease, which occurs in the West Indies and some other tropical and sub-tropical countries, the small hepatic veins are occluded by subendothelial œdema with subsequent collagenization.

The Budd-Chiari syndrome is due to thrombosis of the large hepatic veins, and in many cases it is an incidental post-mortem finding. The hepatic气̊ veins are usually partially occluded at their point 3 of union with the inferior vena cava, thrombosis $\stackrel{\curvearrowright}{\varrho}$ either spreading from the inferior vena cava orc. occurring primarily in their ostia; it is most $\vec{F}$ commonly secondary to some other disease $\operatorname{such}^{\text {की }}$ as pressure or invasion by hepatic neoplasms.

\section{(b) Intra-hepatic Obstruction}

The low pressure gradient between the portal $\stackrel{\square}{\varrho}$ and hepatic veins results in easy obstruction ${ }^{\circ}$ to the portal blood flow. The obstruction may $\vec{\circ}$ be temporary or permanent.

Any condition that causes tension in the capsule, such as acute hepatitis, will probably을 obstruct the portal blood flow temporarily leading 3 . to portal hypertension but the pressure returns to normal when the lesion resolves.

A longer-standing portal hypertension arises $\vec{f}$ in conditions which result in a fibrous response $\vec{\sim}$ in the liver, and hepatic cirrhosis is considered 8 to be the main cause of intra-hepatic obstruction. $\frac{\text { ? }}{2}$ No matter what the cause of the cirrhosis, theultimate histological picture varies little. Portal hypertension in cirrhosis is produced by obstruction of the portal vein branches by fibrosis; by $\vec{\bullet}$ pressure by regenerating liver nodules on the small hepatic veins and by presinusoidal anaste: moses between the hepatic artery and portis vein in the fibrous septa of the cirrhotic liver resulting in an increased hepatic artery blood flow.

The ætiological factors which may give rise to $\frac{}{\varnothing}$ intra-hepatic portal obstruction may be listed $\stackrel{\varrho}{\not}$ as follows:

(1) Virus hepatitis.-Most cases of virus $\frac{3}{3}$ hepatitis resolve completely and do not lead to portal hypertension. It is not known why a few progress to hepatic fibrosis sufficient to lead to the 3 development of portal hypertension. It is thought by some workers that some cases of portal hyper- $\underline{3}$. tension with hepatic fibrosis may be due to a previous unidentified virus hepatitis without jaundice but this cannot be proved until there iso a serological test for virus hepatitis.

(2) Nutritional Deficiency.-There is experi-을 mental evidence that a low protein diet, in spite of an adequate calorie intake, causes diffuse hepatic cell necrosis and post-necrotic scarring. $N$ There is a high incidence of cirrhosis of the liver ${ }_{\mathrm{W}}^{N}$ and portal hypertension in Africa and the Easto and poor nutrition has been blamed for this.o

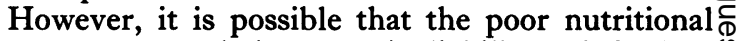
state may merely increase the liability to infection. $\stackrel{?}{?}$

(3) Alcoholism.-It is not known whether $\frac{T}{T}$ alcohol produces the cirrhosis as a direct toxic

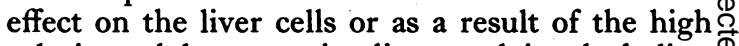
calorie and low protein diet usual in alcoholics. $\stackrel{\mathbb{}}{\varrho}$ 
(4) Hamochromatosis.-Iron accumulates in the liver and leads to fibrosis and portal hypertension.

(5) Hepato-lenticular Degeneration.-In this condition there is an excess of copper in the liver with cirrhosis of post-necrotic or portal type.

(6) Congenital Syphilis. - This very rarely leads to portal hypertension as a result of a fine pericellular cirrhosis with marked fibrous tissue reaction.

(7) Bilharzia.-The ova of Schistosoma mansoni and Schistosoma japonicum obstruct the branches of the portal vein and give rise to periportal fibrosis.

(8) Biliary Cirrhosis.-This may occur in a primary form or be secondary to chronic extrahepatic biliary obstruction such as from gallstones or injury to the common bile duct, and infrequently it may lead to portal hypertension.

(9) Congenital Fibrosis of the Liver.-This is a rare presinusoidal type of obstruction occurring in children. The changes do not appear to be progressive and the only symptom is hæmatemesis.

(I0) Granulomatous Lesions.--Sarcoidosis affects the liver and has been followed by portal hypertension.

(I I) Thickened Liver Capsule.-Cases of portal hypertension have been encountered associated with a very thickened liver capsule which may be calcified in places. These cases may result from massive peripheral necrosis of the liver parenchyma, and traces of portal tracts may be found in the fibrous tissue of the capsule.

(12) Hepatic Neoplasm.-Primary hepatic carcinoma, which sometimes shows calcification, is not uncommon in hepatic cirrhosis. Primary hepatic neoplasms may also occur in children. Metastatic carcinoma in the liver may also on rare occasions lead to portal hypertension and hæmatemesis.

(13) Unknown Atiology.-In about $50 \%$ of the cases of hepatic cirrhosis with portal hypertension no definite ætiology can be found. In many of these cases the pathological condition in the liver is stationary and the only condition that endangers the life of the patient is the portal hypertension.

There are other cases in which there is no extra-hepatic obstruction and in which the liver shows only slight histological variation from normal with no increase in fibrous tissue and with portal tracts either normal or showing only a little cellular infiltration. It is possible that vascular spasm may play a part in obstructing the blood flow through the liver in these cases.

\section{(c) Extra-hepatic Obstruction}

Obstruction of the portal vein may occur in any part of its course and the splenic vein may be involved simultaneously or separately. The obstruction is usually produced by intravascular thrombosis. The possible causes may be listed as follows:

(i) Cirrhosis of the Liver.-In patients who have long standing portal obstruction the veins of the portal system may show intimal lesions and there is slowing or almost complete stasis of the portal blood flow. These factors favour intravascular thrombosis, which may be mural or fill the entire vein. Recanalization may take place in time and there may be calcification in the mural thrombus. Infarction of the small bowel will occur if the thrombus extends to the superior mesenteric vein.

(ii) Portal Phlebitis.-Before the introduction of chemotherapy and antibiotics suppurative phlebitis was almost invariably fatal. Now the infection may be overcome by antibiotic therapy but the legacy will be portal vein thrombosis. Infection anywhere within the drainage area of the portal and splenic veins may cause portal phlebitis, the commonest being the appendix and neonatal umbilical infection.

(iii) Tumours.-Primary or secondary tumours may exert pressure on or invade directly the portal or splenic vein and this may be followed by intravascular thrombosis. The commonest site is in the porta hepatis.

(iv) Blood Disorders.-Any blood disease which carries an increased risk of intravascular thrombosis may cause portal vein thrombosis, e.g. polycythæmia rubra vera.

(v) Trauma.-Rarely portal vein thrombosis or scarring is secondary to injury during operations, particulary cholecystectomy. Portal vein thrombosis also is liable to occur after splenectomy or portocaval anastomosis. However, ligation of the portal vein does not always give rise to manifestations of portal hypertension. External trauma to the upper abdomen may occasionally cause portal vein thrombosis but it is almost impossible to correlate cause and effect.

(vi) Congenital.-It is now recognized that most cases of extra-hepatic portal obstruction, even in young persons, are acquired, and congenital lesions, such as persistent fotal valves in the portal vein producing secondary thrombosis, must be very rare.

\section{Effects of Portal Hypertension}

In time, portal hypertension produces anatomical and functional changes in the splanchnic circulation mainly associated with the development of a collateral circulation and congestive splenomegaly. This occurs where the portal and systemic circulations join and the portal blood is diverted into systemic channels. It develops particularly round the osophagus and the gastric 
fundus, retroperitoneally and in the falciform ligament. The spleen is always enlarged in portal hypertension but its size is not proportional to the degree of elevation of the portal pressure. The enlargement is partly due to congestion but long standing congestion causes structural changes which take time to resolve. The splenic enlargement can give rise to the blood changes of hypersplenism, i.e. anæmia, leucopenia and thrombocytopenia. Probably as the result of infarcts, vascular adhesions may develop between the spleen and the abdominal wall. It is usually but not always palpable.

The only clinical findings due directly to the portal hypertension are associated with the collateral circulation. Patients with intra-hepatic obstruction may also have clinical features of their liver disease which will not be discussed here.

If there are extensive varices up the œsophagus the most serious danger is hæmorrhage which may be a slow ooze leading to anæmia and melæna or more frequently a severe hæmatemesis which comes suddenly and without warning. Less than $10 \%$ of hæmatemeses in patients in Great Britain are due to portal hypertension. However, in Africa and Central and South America and in children in Great Britain, portal hypertension is the commonest cause. The factor which precipitates the hæmorrhage may be peptic ulceration of the varices, trauma from food particles or sudden rise in intra-abdominal or portal pressure. The intervals between hæmorrhages are very variable, ranging from a few days to several years. It is very unusual for a patient to die from his first hæmorrhage but his prognosis is worse than that of the patient bleeding from peptic ulceration. Following hæmorrhage the patient may pass into the state of liver failure or suffer neuropathic symptoms and even pass into coma from absorption of protein products from digestion of the blood in the gut.

\section{The Radiological Investigation of Portal Hypertension}

In cases of sudden hæmorrhage clinical distinction of portal hypertension from peptic ulceration may be difficult.

Straight $X$-ray.-In such cases an overpenetrated view of the chest or tomography when the patient can be moved may be helpful to demonstrate the enlarged azygos and hemiazygos veins and a plain film of the abdomen may reveal splenic enlargement that cannot be palpated. When the patient is fit enough gastric and osophageal varices as well as other possible causes of hæmatemesis should be sought by barium examination.

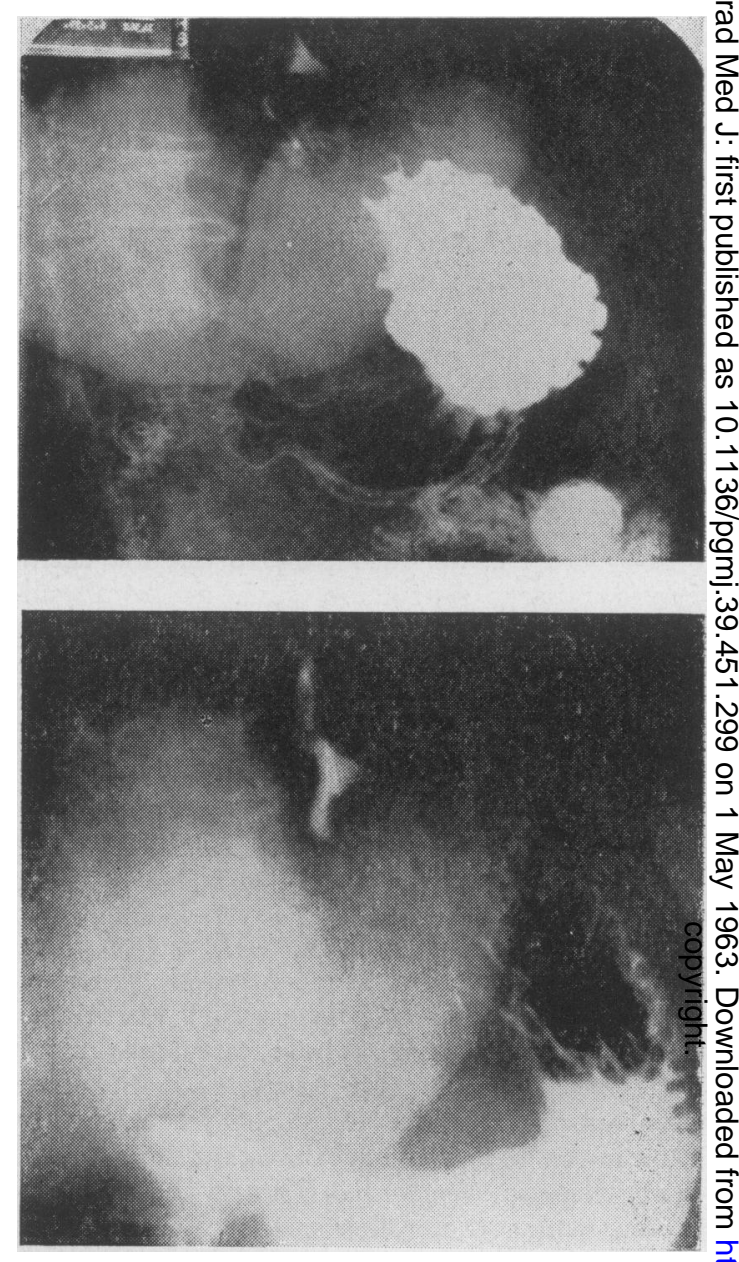

FIG. Ia and Ib.-Supine and erect films showing gastric varices as filling defects in the fundus.

Barium Examination.-The demonstration of a $\frac{5}{3}$ collateral circulation by barium examination of the upper alimentary tract must be the next $\frac{O}{?}$ step. Gastric varices are best outlined by coating the fundus of the stomach with neat barium (the authors prefer Micropaque) by first lying the $\frac{D}{2}$ patient supine and then bringing him into the erect or prone position so that a double barium/ o air contrast of the fundus is produced. So as not to get too much barium in the stomach it is best to start examining the patient by looking for gastric $\omega$ varices. The varices appear as lobulated or rounded filling defects in the fundus and in the region of the upper part of the lesser curve. $\mathbb{D}$ Sometimes they appear as thickening and tortuosity of the mucosal folds in the fundus. On other $\frac{T}{\circ}$ occasions they are so large that they present as a $\frac{\vec{\Phi}}{\mathbb{P}}$ large soft tissue filling defect on the medial side $\frac{\rho}{\mathbb{D}}$ which will be apparent against the air in the $\varrho$ 


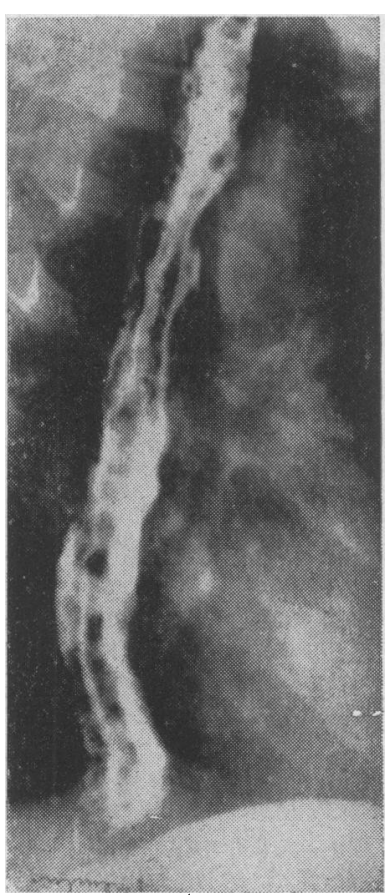

FIG. 2.-Esophageal varices.

gastric fundus or cause a smooth lobulated filling defect in the barium-filled fundus in the supine position. In these cases the mass of varices has to be distinguished from a carcinoma (Fig. I).

Esophageal varices will only be demonstrated on good mucosal pattern films of the lower end of the csophagus. Neat Micropaque is used and if this is unsuccessful, due to non-adhesion of the barium, Microtrast is then used. Small varices will only show in the relaxed œsophagus and care must be taken to see that the radiographs are not exposed when a contraction wave is present or when the cesophagus is full of barium, as these features will obliterate varices. The least useful position is the erect. The best position is the prone, with the left side raised and after that the supine position with the right side raised. The respiratory phase should be midway between inspiration and expiration. The Valsalva and Muller manœuvres are occasionally useful for bringing out the varices, but in most cases they are unhelpful. Small varices produce some thickening of, and small rounded projections on, the borders of the mucosal folds at the lower end of the œsophagus and also filling defects on the cesophageal outline. Large varices produce linear, globular or worm-like filling defects of varying size and are associated with dilatation of the cesophagus and complete distortion of the mucosal pattern of the relaxed œsophagus (Fig. 2).
Varices may just involve the lower end of the cesophagus or they may extend right up into the neck. Bubbles of air may simulate varices. They are, however, more translucent than varices, not constant and usually facetted. The accuracy of barium examination in the demonstration of varices is difficult to determine as there is reluctance of many surgeons to pass an œsophagoscope on these patients. Barium studies, of course, will only demonstrate submucosal varices and csophagoscopy is the only practical method of inspecting the œsophageal mucosa. The difficulty always is to demonstrate small varices, as large varices are usually obvious. Different workers claim a varying accuracy of demonstration of known œsophageal varices from about $14 \%$ to $87 \%$. Selection of cases probably accounts largely for the very differing accuracy figures. There are other difficulties in the detection of varices. It has been established that the dimensions of varices vary from time to time with spontaneous fluctuations in portal pressure and with changes in blood volume, such as occur after a hæmorrhage. Occasionally large gastric varices are seen radiologically without the presence of œsophageal varices. This may be due to the peculiarities of the collateral circulation with drainage of the gastric varices into the retroperitoneal veins or in cases of splenic vein thrombosis, drainage back from the fundus into a left gastric vein entering directly into a patent portal vein and not into the thrombosed splenic vein. For practical purposes the demonstration of varices by radiological or œsophagoscopy methods can be taken as definite evidence of appreciable portal hypertension. There are cases, however, with large collateral channels in which the portal pressure is normal or near normal. In these cases it is thought that the size of the collateral channels has lowered the peripheral resistance of the portal circulation sufficiently to lower the pressure.

Portal Venography.-The object of portal venography is to demonstrate the state of the splenic and portal veins, the site of an obstruction and the presence or absence of thrombi in these veins so that the optimal surgical approach can be planned. The fact that portal hypertension is present is determined by the prior measurement of the intrasplenic pressure, but the showing of a large collateral circulation in cases with near normal pressures indicates appreciable obstruction in the outflow from the liver.

Trans-splenic Portal Venography.-Provided splenectomy has not previously been performed this examination is performed first, by percutaneous splenic puncture. This is done under local anæsthetic in adults: a general anæsthetic is given 
to children. Occasionally a general anæsthetic is required with adults, and it is done by this method in the operating theatre when it is desired to go straight on to a shunt operation, particularly in patients who have had a recent severe hæmatemesis. In adults it is very important to explain carefully to the patient every step of the examination so that their full co-operation is secured. Particularly is it explained that once the needle is in position they must breath only very shallowly while the intra-splenic pressure is being measured, and that when they are told to stop breathing at the time of the injection and taking of the films, they must hold their breath in whatever phase of respiration they are at that time. Some patients tend to take a huge inspiration before stopping breathing. This phenomenon may account for the occasional cases where varices are shown at barium swallow and not demonstrated by portal venography. Premedication is by $75 \mathrm{mg}$. pethidine intramuscularly. A test dose of the contrast medium is given in spite of the known limitations of its efficiency in detecting sensitivity reactions. A control film is taken to check the patient's position and the radiographic factors. Two $17 / 14$ films are placed transversely in a modified PotterBucky tray, the top film being lead backed to protect the lower one from radiation. The films are so positioned that the top of the iliac crest just appears on the bottom of the film and this allows coverage of the lower part of the œsophagus. The X-ray tube column is offcentred from the Bucky tray so that access to the left side of the patient is not impeded. This method has been found quite adequate for this examination in the hands of the present writers, though in other schools a serial changer is used. The spleen is punctured either below the costal margin, if it is large enough, or in the intercostal space in line with the apex of the spleen if it is only just palpable, i.e. the 8th or 9th interspace. If the spleen is impalpable it is punctured over the area of maximum dullness. The needle is a 17 S.W.G. needle and it is inserted through the abdominal wall down to the spleen in a direction upwards, backwards and medially and the resistance as it enters the spleen can be felt. It is then advanced a centimetre or so and blood dripping slowly from it indicates that it is correctly sited. A saline pressure manometer is attached and the intrasplenic pressure recorded. The syringe, containing $30 \mathrm{ml} .85 \%$ Hypaque warmed to $120^{\circ} \mathrm{F}$, is now connected to the needle and the patient is instructed to hold his breath in a midrespiratory phase. The injection is made rapidly by hand and the first film taken two thirds of the way through the injection and the second about three seconds later. The Bucky is recharged after

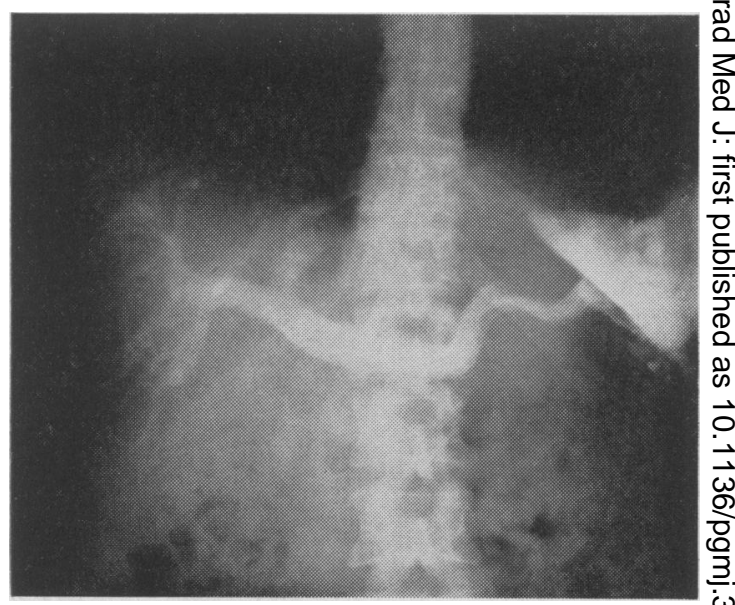

FIG. 3.-Normal portal venogram showing splenic vein and portal vein, the latter branching within the $\theta$ liver. The streaming effect due to non-opaque blood from the superior mesenteric vein can be seen. î

the first film has been taken. The needle is then 을 withdrawn and the patient allowed to breath again. -

The patient's immediate reactions are a good indication of the success or failure of the examination. If they experience a feeling of warmth $\vec{\odot}$ across the upper abdomen, the contrast medium $\omega$ has been successfully injected into the spleent and has crossed the upper abdomen in the veins. If they experience local left-sided pain during the injection and particularly if it radiates to the tip of the left shoulder some of the contrast medium $\Phi$ has run into the peritoneum around the undersurface of the diaphragm. This pain is not $\frac{3}{3}$ usually severe and it is accentuated by respiration. $\frac{}{3}$ If there is no immediate pain but severe local left-sided pain develops a few minutes later, the injection has gone under the splenic capsule, and $\frac{3}{3}$ if there is pain down the left side of the abdomen $:$ into the pelvis the contrast medium has tracked 3 . down the left paracolic gutter. Should the $\delta$ examination be a failure it is never repeated on $₹$ the same day. If there are no immediate contrast 0 medium reactions the patient is returned to the ward and kept on a half-hourly pulse chart for three hours. Apart from the dangers of injecting the contrast medium into any patient the particular or dangers of trans-splenic portal venography are $N$ hæmorrhage either into the spleen itself or into $\mathrm{N}_{\mathrm{J}}$ the peritoneum from tear of the spleen at the $\sigma$ puncture site. In most cases intraperitoneal hæmorrhage is minimal and undetectable, but $\frac{\bar{D}}{D}$ occasionally a small quantity of blood may be $\stackrel{\leftrightarrow}{?}$ found in the peritoneum at laparotomy a few 0 days later.

Operative Portal Venography.-This is per- $\frac{\mathbb{Q}}{\overparen{D}}$ formed if the patient has had a previous splenec- 


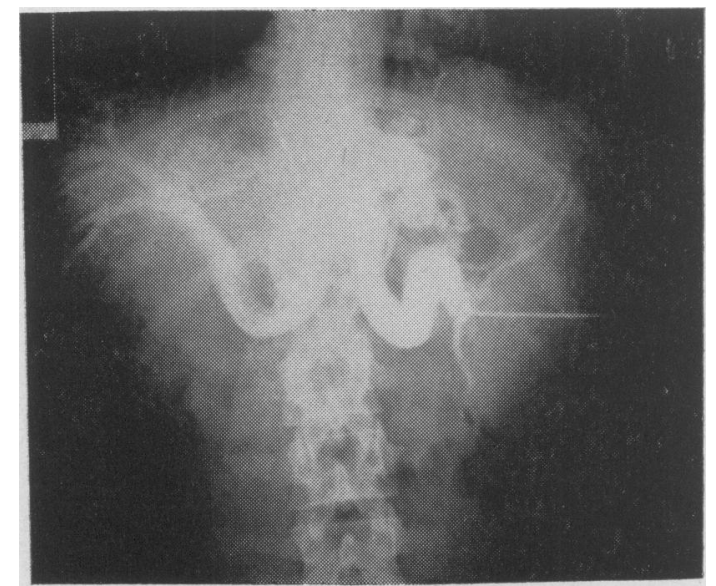

FIG. 4.-Portal venogram in intra-hepatic obstruction. The splenic vein is tortuous and there are collateral channels through the left gastric vein and gastric varices. The portal vein is of normal calibre.

tomy or if for any reason the portal vein has not been demonstrated prior to laparotomy. The anterior end of the right thoraco-abdominal incision used for porto-caval anastomosis is made first and the same vein is used which has been cannulated for measuring the portal venous pressure. The anæsthetist orders the patient to stop breathing, 20 to $30 \mathrm{ml} .85 \%$ Hypaque is injected, and two 17/14 fast films are taken, the first two thirds of the way through the injection and the second as soon after the injection as possible, using a simple cassette tunnel and a mobile X-ray unit.

The contraindications to portal venography are considered to be:

(I) Age: Porto-caval anastomoses are not usually performed on patients over the age of 60 years so, in general, advanced age is a contraindication.

(2) Jaundice.

(3) A prothrombin time less than $75 \%$ of normal.

(4) Inadequate number of blood platelets, i.e. less than $100,000 / \mathrm{cu} . \mathrm{mm}$.

\section{Radiological Findings in Portal Venography}

(i) Normal Appearances.-Normal portal venograms are not often obtained (Fig. 3). From the pool of contrast medium in the splenic pulp one or more intrasplenic branches of the splenic vein may be outlined. These converge on the hilum to form the main splenic vein which forms a slight curve as it passes from left to right horizontally across the upper abdomen. To the right of, or in front of the body of the second lumbar vertebra it joins the portal vein. This vein passes upwards to the right for a short distance and divides into the left and right branches, occasionally with a middle branch. These branches divide again into smaller branches within the liver usually by simple forking, decreasing in size as they pass to the periphery of the liver. The branches of the left lobe can be seen passing to the left below the diaphragm. When the contrast medium leaves the larger branches in the liver it then enters the sinusoids, at which time the liver is seen to be diffusely opaque, i.e. a hepatogram is obtained. This gradually clears. Where the superior mesenteric vein joins the splenic vein a streaming effect is usually produced by the non-opaque blood entering from the superior mesenteric vein, causing a less opaque stream in the centre of the left half of the portal vein which gradually merges with the right part of the vein. A similar streaming effect is seen in the splenic vein from its branches in the spleen.

(ii) Appearances of Intra-hepatic Obstruction.The plain film will show splenomegaly which may assume various shapes. The spleen may be oval or elongated or it may be tucked up under the diaphragm. The stomach gas shadow is displaced to the right and occasionally linear calcification may be seen in the portal, splenic or superior mesenteric veins. On the portal venogram the splenic and portal veins are patent and usually wide. The splenic vein exhibits various degrees of tortuosity depending on the amount of shift of the splenic hilum to the right. Various collateral pathways will be shown by retrograde flow of the contrast medium along them (Fig. 4). Usually the left gastric vein fills and varices in the region of the upper part of the lesser curve of the stomach are outlined. These may be small and numerous or few and very large. Varices from the fundus of the stomach may also be filled from the short gastric veins. The contrast medium then passes above the diaphragm in front of and on either side of the thoracic spine as osophageal varices are filled. Several smaller gastric varices may be filled from the upper border of the splenic vein. Occasionally a large para-umbilical vein from the left branch of the portal vein may be filled. This passes downwards and to the left towards the lower abdomen. Superior and inferior mesenteric veins may also be filled retrogradely as well as capsular veins round the spleen and vessels in the vascular adhesions to the lateral and posterior abdominal wall. Through these latter channels the inter-costal veins and anastomoses through the lumber and left renal veins may lead to filling of the inferior vena cava. Distortion of the portal radicles within the liver may also be seen. Occasionally a large omental vein is filled, draining 


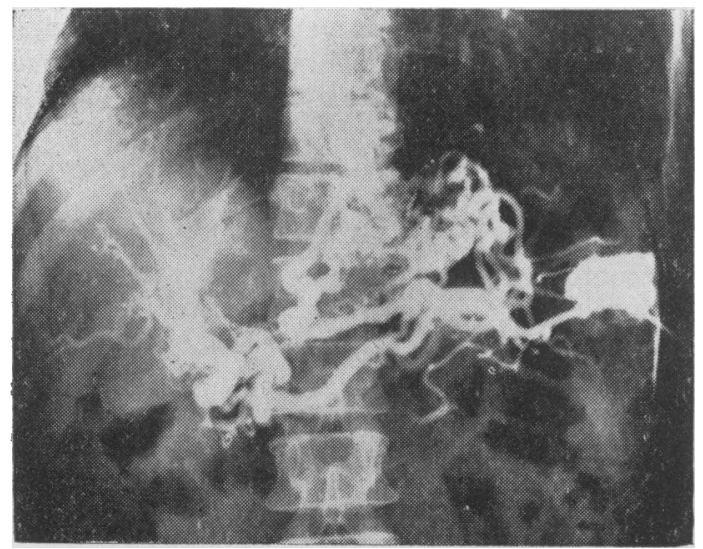

FIG. 5.-Portal venogram in extra-hepatic obstruction at the entrance of the portal vein into the liver.

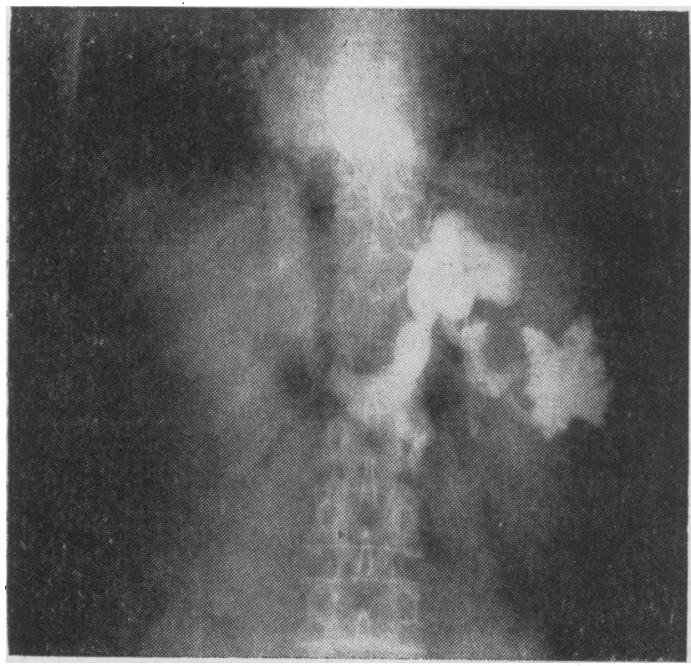

FIG. 7.- - Portal venogram in extra-hepatic obstruction in the splenic vein. Large, tortuous, varicosities are shown crossing the abdomen and a connection with the inferior vena cava has been established.

to some part of the lateral abdominal wall. Thrombi in the portal and splenic veins will show either as complete obstruction of the vein, diffuse narrowing of the vein or as a localized marginal filling defect. If the thrombus is marginal on the anterior or posterior wall of the vein it will show as a central or near-central filling defect which may be elongated giving an appearance rather like streaming, but it is constant on serial films in comparison with true streaming which will vary its appearance and be distal to the entrance of a large vein. A number of thrombi in the portal vein do not show up by portal venography, but such thrombi do not preclude a porto-caval anastomosis being performed as they can be removed at operation. Extrinsic pressure on the portal vein may be caused

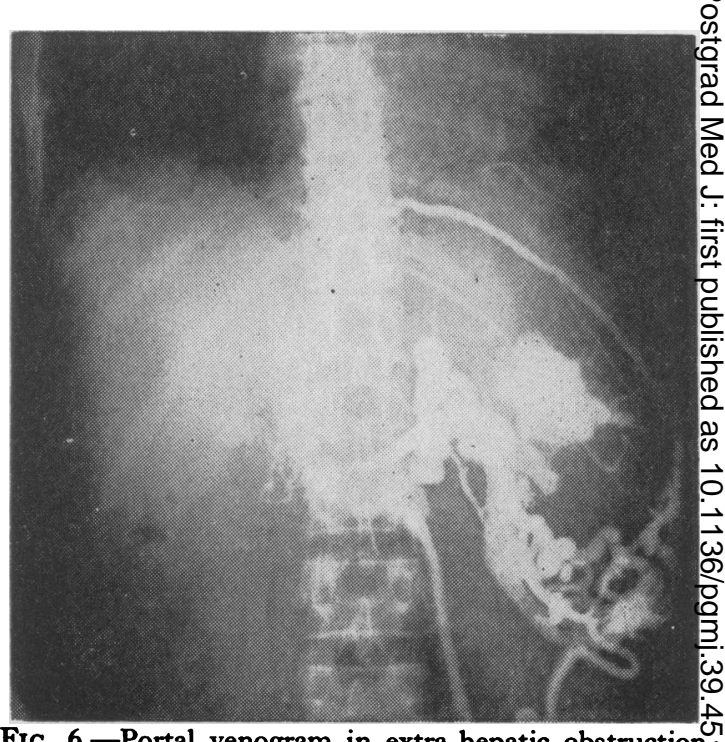

Fig. 6.-Portal venogram in extra-hepatic obstructionwhere the block affects all the portal vein. Multiplei collateral channels are shown.

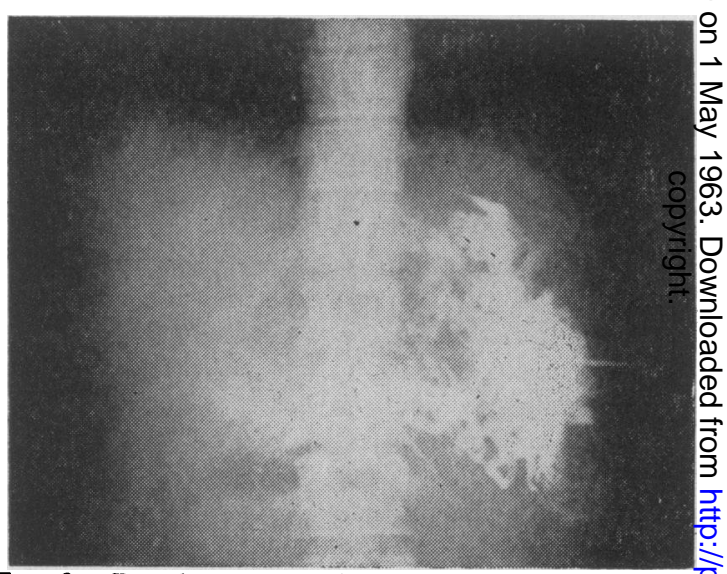

Fig. 8.-Portal venogram in extra-hepatic obstruction where the block affects the splenic vein.

by other structures, such as choledochal cyst.

(iii) Extra-hepatic Obstruction.-The appear-O ances will vary with the site of obstruction.

(a) At the Entrance of the Portal Vein into thes Liver.-The splenic and portal veins are patent but the portal vein is completely obstructed at the extreme hepatic end. A mass of small veins $N$ surround the site of the obstruction and they extend into the liver where the intra-hepatic branches may be poorly filled. This irregular net- $\omega$ work of para-portal veins of varying size gives an? appearance like a venous angioma (Fig. 5). This has led to the supposition that this meshwork of veins is a congenital abnormality, the so-called ${ }^{+}$ cavernomatous malformation of the portal vein. $\frac{T}{0}$ The extent of its range will depend on the extent $\frac{\vec{D}}{\mathrm{D}}$ of the original obstruction. The resistance to $\frac{\rho}{\mathbb{D}}$ 
the flow of the blood through these tortuous channels must be very great as the portal venous pressure is frequently above $30 \mathrm{~mm}$. $\mathrm{Hg}$. Collaterals over the surface of the liver may be shown and if the right branch only is obstructed there may be filling of a large para-umbilical vein.

(b) All the Portal vein.-In such cases only the splenic vein and collateral channels are filled (Fig. 6). The portal vein may be bypassed by para-portal collaterals which may be few and fairly large, or a mass of very small veins. The contrast medium is frequently sidetracked down one or two large collaterals, such as the left gastric vein, inferior mesenteric vein or through the retroperitoneal collaterals to the left renal vein and into the inferior vena cava (see Fig. 7).

(c) Splenic and Portal Veins.-No contrast medium enters the portal or splenic vein and a mass of collaterals are shown usually crossing the abdomen towards the porta hepatis (Figs. 7 and 8). These represent pancreatic veins. The collaterals may extend up and down the left side of the lumbar spine and in which case they are usually veins of the retroperitoneal tissues in the lienorenal ligament and lumbar veins. Inter-costal and diaphragmatic veins may also be filled.

(d) False Extra-hepatic Obstruction.-If the pressure in the portal vein or splenic vein is very high, presumably due to higher sinusoidal pressure or large arterio-venous shunts, there may be reversal of blood flow in the portal and splenic veins. In these cases the contrast medium injected into the spleen will not enter the splenic or portal veins and will be diverted down large collateral channels. However, if the contrast medium is injected into a jejunal vein at laparotomy, the splenic and portal veins will be demonstrated. It is for this reason that operative portal venography is always carried out on patients whose portal and splenic veins are not filled on the transsplenic route.

\section{Discussion}

The main operations for portal hypertension have one of the following objects:

(I) To reduce portal-venous pressure to safe limits by wide venous anastomosis.

(2) To cut off the flow of portal blood to bleeding varices.

(3) Reduction of flow through the liver.

The only veins of the portal circulation available for anastomosis to veins of the systemic circulation are the portal and splenic veins. Porto-caval anastomosis is performed if the obstruction is in the liver. Spleno-renal anastomosis can be performed if the portal vein is occluded. Portocaval anastomosis is performed either by anastomosing the end of the portal vein to the side of the inferior vena cava or by side-to-side anastomosis of the portal vein to the inferior vena cava. These are the only operations which reduce the portal blood pressure.

If there are no suitable veins for anastomosis gastric or asophageal transection may be carried out, experienced surgeons now favouring the latter, in which, through a left thoracotomy incision, the lower end of the cesophagus is divided longitudinally through the muscle coat and the mucosa divided and re-sutured, any vessels on the surface of the œsophagus being tied.

The most serious complication of a portocaval anastomosis is portal systemic encephalopathy, which may even arise in patients who have not had such an anastomosis performed and who have a large portal systemic collateral circulation.

It has been shown that some of the neurological symptoms which occur in cases of liver disease are due to shunting of portal blood directly into the systemic circulation whence it reaches the brain before it has passed through the liver. Porto-caval anastomosis produces such a shunt and cases would be expected to arise after it. There is no doubt that the condition is due to some protein breakdown product containing nitrogen entering the systemic circulation. The encephalopathy manifests itself in a variety of ways but in the same patient each episode tends to hold to a constant pattern. The commonest symptom is increasing drowsiness passing into coma, but some patients may pass very suddenly from an apparently normal state to complete unconsciousness and equally suddenly return to their earlier condition.

In the hands of some surgeons the operative treatment of portal hypertension, either by portocaval anastomosis or if this is not possible, by œsophageal transection, has been very successful. Recurrent hæmatemesis after porto-caval anastomosis is rare, and its incidence after œsophageal transection greatly reduced.

In the initial investigation of possible cases of portal hypertension and in the subsequent detailed pre-operative investigation of such cases, radiological procedures have an important and essential part to play. These procedures carry no great risk, but if they are to be of value it is essential that they should be carried out by an experienced team and with very great consideration to every detail of the procedures.

\section{Summary}

The anatomy of the portal circulation is described and the pathology of portal hypertension discussed. Radiological procedures for the investigation of such cases are then considered and the appearances described in detail. 